Advances in Fuzzy Mathematics.

ISSN 0973-533X Volume 12, Number 3 (2017), pp. 515-538

(C) Research India Publications

https://dx.doi.org/10.37622/AFM/12.3.2017.515-538

\title{
Multi-Objective Welded Beam Optimization using Neutrosophic Goal Programming Technique
}

\author{
Mridula Sarkar* \\ Department of Mathematics, Indian Institute of Engineering Science and Technology, \\ Shibpur, P.O.-Botanic Garden, Howrah-711103, West Bengal, India. \\ Tapan Kumar Roy \\ Department of Mathematics, Indian Institute of Engineering Science and Technology, \\ Shibpur, P.O.-Botanic Garden, Howrah-711103, West Bengal, India. \\ *corresponding author
}

\begin{abstract}
This paper investigates multi-objective Neutrosophic Goal Optimization (NSGO) approach to optimize the cost of welding and deflection at the tip of a welded steel beam, while the maximum shear stress in the weld group, maximum bending stress in the beam, and buckling load of the beam have been considered as constraints. The problem of designing an optimal welded beam consists of dimensioning a welded steel beam and the welding length so as to minimize its cost, subject to the constraints as stated above. The classical welded bream design structure is presented here in to demonstrate the efficiency of the neutrosophic goal programming approach. The model is numerically illustrated by generalized NSGO technique with different aggregation method. The result shows that the Neutrosophic Goal Optimization technique is very efficient in finding the best optimal solutions.
\end{abstract}

Keyword: Neutrosophic Set, Single Valued Neutrosophic Set, Generalized Neutrosophic Goal Programming, Arithmatic Aggreegation ,Geometric Aggreegation, Welded Beam Design Optimization .

\section{INTRODUCTION}

Welding, a process of joining metallic parts with the application of heat or pressure or the both, with or without added material, is an economical and efficient method for 
obtaining permanent joints in the metallic parts. This welded joints are generally used as a substitute for riveted joint or can be used as an alternative method for casting or forging. The welding processes can broadly be classified into following two groups, the welding process that uses heat alone to join two metallic parts and the welding process that uses a combination of heat and pressure for joining (Bhandari. V. B). However, above all the design of welded beam should preferably be economical and durable one. Since decades, deterministic optimization has been widely used in practice for optimizing welded connection design. These include mathematical optimization algorithms (Ragsdell \& Phillips 1976) such as APPROX (Griffith \& Stewart's) successive linear approximation, DAVID (Davidon Fletcher Powell with a penalty function), SIMPLEX (Simplex method with a penalty function), and RANDOM (Richardson's random method) algorithms, GA-based methods (Deb 1991, Deb 2000, Coello 2000b, Coello 2008), particle swarm optimization (Reddy 2007), harmony search method (Lee \& Geem 2005), and Big-Bang Big-Crunch (BBBC) (O. Hasançebi, 2011) algorithm. SOPT (O. Hasançebi, 2012), subset simulation (Li 2010), improved harmony search algorithm (Mahadavi 2007), were other methods used to solve this problem. Recently a robust and reliable $H \infty$ static output feedback (SOF) control for nonlinear systems (Yanling Wei 2016) and for continuous-time nonlinear stochastic systems (Yanling Wei 2016) with actuator fault in a descriptor system framework have been studied. All these deterministic optimizations aim to search the optimum solution under given constraints without consideration of uncertainties. So, while a deterministic optimization approach is unable to handle structural performances such as imprecise stresses and deflection etc. due to the presence of uncertainties, to get rid of such problem fuzzy (Zadeh, 1965), intuitionistic fuzzy (Atanassov,1986), Neutrosophic (Smarandache,1995) play great roles.

Traditionally structural design optimization is a well known concept and in many situations it is treated as single objective form, where the objective is known the weight or cost function. The extension of this is the optimization where one or more constraints are simultaneously satisfied next to the minimization of the weight or cost function. This does not always hold good in real world problems where multiple and conflicting objectives frequently exist. In this consequence a methodology known as multi-objective optimization (MOSO) is introduced

So to deal with different impreciseness such as stresses and deflection with multiple objective, we have been motivated to incorporate the concept of neutrosophic set in this problem, and have developed multi-objective neutrosophic optimization algorithm to optimize the optimum design.

Usually Intuitionistic fuzzy set, which is the generalization of fuzzy sets, considers both truth membership and falsity membership that can handle incomplete information excluding the indeterminate and inconsistent information while neutrosophic set can quantify indeterminacy explicitly by defining truth, indeterminacy and falsity membership function independently. Therefore, Wang et.al (2010) presented such set as single valued neutrosophic set (SVNS) as it comprised of 
generalized classic set, fuzzy set, interval valued fuzzy set, intuitionistic fuzzy set and Para-consistent set.

As application of SVNS optimization method is rare in welded beam design, hence it is used to minimize the cost of welding by considering shear stress, bending stress in the beam, the buckling load on the bar, the deflection of the beam as constraints. Therefore the result has been compared among three cited methods in each of which impreciseness has been considered completely in different way.

Moreover using above cited concept, a multi-objective neutrosophic optimization algorithm has been developed to optimize three bar truss design (Sarkar 2016), and to optimize riser design problem (Das 2015). In early 1961 Charnes and Cooper first introduced Goal programming problem for a linear model. Usually conflicting goal are presented in a multi-objective goal programming problem. Dey et al.(2015) used intuitionistic goal programming on nonlinear structural model.

However, the factors governing of former constraints are height and length of the welded beam, forces on the beam, moment of load about the centre of gravity of the weld group, polar moment of inertia of the weld group respectively. While, the second constraint considers forces on the beam, length and size of the weld, depth and width of the welded beam respectively. Third constraint includes height and width of the welded beam. Fourth constraints consists of height, length, depth and width of the welded beam. Lastly fifth constraint includes height of the welded beam. Besides, flexibility has been given in shear stress, bending stress and deflection only, hence all these parameters become imprecise in nature so that it can be considered as neutrosophic set to from truth, indeterminacy and falsity membership functions Ultimately, neutrosophic optimization technique has been applied on the basis of the cited membership functions and outcome of such process provides the minimum cost of welding ,minimum deflection for nonlinear welded beam design. The comparison of results shows difference between the optimum value when partially unknown information is fully considered or not. This is the first time NSGO technique is in application to multi-objective welded beam design. The present study investigates computational algorithm for solving multi-objective welded beam problem by single valued generalized NSGO technique. The results are compared numerically for different aggregation method of NSGO technique. From our numerical result, it has been seen that the best result obtained for geometric aggregation method for NSGO technique in the perspective of structural optimization technique.

\section{MULTI-OBJECTIVE STRUCTURAL MODEL}

In sizing optimization problems, the aim is to minimize multi objective function, usually the cost of the structure, deflection under certain behavioural constraints which are displacement or stresses. The design variables are most frequently chosen to be dimensions of the height, length, depth and width of the structures. Due to fabrications limitations the design variables are not continuous but discrete for 
belongingness of cross-sections to a certain set. A discrete structural optimization problem can be formulated in the following form

Minimize $C(X)$

Minimize $\delta(X)$

subject to $\sigma_{i}(X) \leq\left[\sigma_{i}(X)\right], i=1,2, \ldots ., m$

$X_{j} \in R^{d}, \quad j=1,2, \ldots . ., n$

where $C(X), \delta(X)$ and $\sigma_{i}(X)$ as represent cost function, deflection and the behavioural constraints respectively whereas $\left[\sigma_{i}(X)\right]$ denotes the maximum allowable value , ' $m$ ' and ' $n$ ' are the number of constraints and design variables respectively. A given set of discrete value is expressed by $R^{d}$ and in this paper objective functions are taken as

$C(X)=\sum_{t=1}^{T} c_{t} \prod_{n=1}^{m} x_{n}^{t n}$ and $\delta(X)$

and constraint are chosen to be stress of structures as follows

$\sigma_{i}(A) \lesssim \sigma_{i}$ with allowable tolerance $\sigma_{i}^{0}$ for $i=1,2, \ldots, m$

Where $c_{t}$ is the cost coefficient of $\mathrm{t}^{\text {th }}$ side and $x_{n}$ is the $n^{\text {th }}$ design variable respectively, $m$ is the number of structural element, $\sigma_{i}$ and $\sigma_{i}^{0}$ are the $i^{\text {th }}$ stress , allowable stress respectively..

\section{MATHEMATICAL PRELIMINARIES}

\subsection{Fuzzy Set}

Let $X$ be a fixed set. A fuzzy set $A$ set of $X$ is an object having the form $\tilde{A}=\left\{\left(x, T_{A}(x)\right): x \in X\right\}$ where the function $T_{A}: X \rightarrow[0,1]$ defined the truth membership of the element $x \in X$ to the set $A$.

\subsection{Intuitionistic Fuzzy Set}

Let a set $X$ be fixed. An intuitionistic fuzzy set or IFS $\tilde{A}^{i}$ in $X$ is an object of the form $\tilde{A}^{i}=\left\{<X, T_{A}(x), F_{A}(x)>\mid x \in X\right\}$ where $T_{A}: X \rightarrow[0,1]$ and $\quad F_{A}: X \rightarrow[0,1]$ 
define the truth membership and falsity membership respectively,for every element of $x \in X, 0 \leq T_{A}(x)+F_{A}(x) \leq 1$.

\subsection{Neutrosophic Set}

Let a set $X$ be a space of points (objects) and $x \in X$.A neutrosophic set $\tilde{A}^{n}$ in $X$ is defined by a truth membership function $T_{A}(x)$, an indeterminacy-membership function $I_{A}(x)$ and a falsity membership function $F_{A}(x)$ and having the form $\tilde{A}^{n}=\left\{<x, T_{A}(x), I_{A}(x), F_{A}(x)>\mid x \in X\right\} . T_{A}(x), I_{A}(x)$ and $F_{A}(x)$ are real standard or non-standard subsets of $] 0^{-}, 1^{+}[$. That is

$\left.T_{A}(x): X \rightarrow\right] 0^{-}, 1^{+}[$

$\left.I_{A}(x): X \rightarrow\right] 0^{-}, 1^{+}[$

$\left.F_{A}(x): X \rightarrow\right] 0^{-}, 1^{+}[$

There is no restriction on the sum of $T_{A}(x), I_{A}(x)$ and $F_{A}(x)$ so $0^{-} \leq \sup T_{A}(x) \leq \sup I_{A}(x) \leq \sup F_{A}(x) \leq 3^{+}$.

\subsection{Single Valued Neutrosophic Set}

Let a set $X$ be the universe of discourse. A single valued neutrosophic set $\tilde{A}^{n}$ over $X$ is an object having the form $\tilde{A}^{n}=\left\{<x, T_{A}(x), I_{A}(x), F_{A}(x)>\mid x \in X\right\}$ where $T_{A}: X \rightarrow[0,1], I_{A}: X \rightarrow[0,1]$ and $F_{A}: X \rightarrow[0,1]$ with $0 \leq T_{A}(x)+I_{A}(x)+F_{A}(x) \leq 3$ for all $x \in X$.

\subsection{Single Valued Generalized Neutrosophic Set}

Let a set $X$ be the universe of discourse. A single valued neutrosophic set $\tilde{A}^{n}$ over $X$ is an object having the form $\tilde{A}^{n}=\left\{<x, T_{A}(x), I_{A}(x), F_{A}(x)>\mid x \in X\right\}$ where $T_{A}: X \rightarrow\left[0, w_{1}\right], I_{A}: X \rightarrow\left[0, w_{2}\right]$ and $\quad F_{A}: X \rightarrow\left[0, w_{3}\right]$ with $0 \leq T_{A}(x)+I_{A}(x)+F_{A}(x) \leq w_{1}+w_{2}+w_{3}$ where $w_{1}, w_{2}, w_{3} \in[0,1]$ for all $x \in X$.

\subsection{Complement of Neutrosophic Set}

Complement of a single valued neutrosophic set $A$ is denoted by $c(A)$ and is defined

$$
\begin{aligned}
T_{c(A)}(x) & =F_{A}(x) \\
\text { by } I_{c(A)}(x) & =1-F_{A}(x) \\
F_{c(A)}(x) & =T_{A}(x)
\end{aligned}
$$




\subsection{Union of Neutrosophic Sets}

The union of two single valued neutrosophic sets $A$ and $B$ is a single valued neutrosophic set $C$, written as $C=A \cup B$, whose truth membership, indeterminacymembership and falsity-membership functions are given by

$$
\begin{aligned}
& T_{c(A)}(x)=\max \left(T_{A}(x), T_{B}(x)\right) \\
& I_{c(A)}(x)=\max \left(I_{A}(x), I_{B}(x)\right) \\
& F_{c(A)}(x)=\min \left(F_{A}(x), F_{B}(x)\right) \text { for all } x \in X
\end{aligned}
$$

\subsection{Intersection of Neutrosophic Sets}

The intersection of two single valued neutrosophic sets $A$ and $B$ is a single valued neutrosophic set $C$, written as $C=A \cap B$, whose truth membership, indeterminacymembership and falsity-membership functions are given by

$$
\begin{aligned}
& T_{c(A)}(x)=\min \left(T_{A}(x), T_{B}(x)\right) \\
& I_{c(A)}(x)=\min \left(I_{A}(x), I_{B}(x)\right) \\
& F_{c(A)}(x)=\max \left(F_{A}(x), F_{B}(x)\right) \text { for all } x \in X
\end{aligned}
$$

\section{MATHEMATICAL ANALYSIS}

\subsection{Neutrosophic Goal Programming}

Goal programming can be written as

Find $x=\left(x_{1}, x_{2}, \ldots, x_{n}\right)^{T}$

to achieve:

$z_{i}=t_{i} i=1,2, \ldots, k$

Subject to $x \in X$ where $t_{i}$ are scalars and represent the target achievement levels of the objective functions that the decision maker wishes to attain provided, $X$ is feasible set of constraints.

The nonlinear goal programming problem can be written as

Fin $x=\left(x_{1}, x_{2}, \ldots, x_{n}\right)^{T}$

So as to

Minimize $z_{i}$ with target value $t_{i}$, acceptance tolerance $a_{i}$, indeterminacy tolerance $d_{i}$ rejection tolerance $c_{i}$

$x \in X$

$g_{j}(x) \leq b_{j}, j=1,2, \ldots ., m$

$x_{i} \geq 0, i=1,2, \ldots ., n$ with truth-membership, indeterminacy-membership and falsitymembership functions 


$$
\begin{aligned}
& T_{i}^{1}\left(z_{i}\right)=\left\{\begin{array}{cc}
1 & \text { if } z_{i} \leq t_{i} \\
\left(\frac{t_{i}+a_{i}-z_{i}}{a_{i}}\right) & \text { if } t_{i} \leq z_{i} \leq t_{i}+a_{i} \\
0 & \text { if } z_{i} \geq t_{i}+a_{i}
\end{array}\right. \\
& I_{i}^{1}\left(z_{i}\right)=\left\{\begin{array}{cc}
\left(\frac{z_{i}-t_{i}}{d_{i}}\right) & \text { if } z_{i} \leq t_{i}
\end{array}\right. \\
& \left(\begin{array}{cc}
\left(\frac{t_{i}+a_{i}-z_{i}}{a_{i}-d_{i}}\right) & \text { if } t_{i}+d_{i} \leq z_{i} \leq t_{i}+a_{i}+a_{i} \\
0 & \text { if } z_{i} \geq t_{i}+a_{i}
\end{array}\right. \\
& F_{i}^{1}\left(z_{i}\right)=\left\{\begin{array}{c}
\text { if } z_{i} \leq t_{i} \\
\left(\frac{z_{i}-t_{i}}{c_{i}}\right) \text { if } t_{i} \leq z_{i} \leq t_{i}+c_{i}
\end{array}\right. \\
& \text { if } z_{i} \geq t_{i}+c_{i}
\end{aligned}
$$

To maximize the degree of acceptance and indeterminacy of nonlinear goal programming (NGP) objectives and constraints also to minimize degree of rejection of of NGP objectives and constraints ,

Maximize $T_{z_{i}}\left(z_{i}\right), i=1,2, \ldots ., k$

Maximize $I_{z_{i}}\left(z_{i}\right), i=1,2, \ldots, k$

Minimize $F_{z_{i}}\left(z_{i}\right), i=1,2, \ldots ., k$

Subject to

$0 \leq T_{z_{i}}\left(z_{i}\right)+I_{z_{i}}\left(z_{i}\right)+F_{z_{i}}\left(z_{i}\right) \leq 3, i=1,2, \ldots ., k$

$T_{z_{i}}\left(z_{i}\right) \geq 0, I_{z_{i}}\left(z_{i}\right) \geq 0, F_{z_{i}}\left(z_{i}\right) I=1,2, \ldots, k$

$T_{z_{i}}\left(z_{i}\right) \geq I_{z_{i}}\left(z_{i}\right), I=1,2, \ldots, k$

$T_{z_{i}}\left(z_{i}\right) \geq F_{z_{i}}\left(z_{i}\right), i=1,2, \ldots, k$

$g_{j}(x) \leq b_{j}, j=1,2, \ldots ., m$

$x_{i} \geq 0, i=1,2, \ldots ., n$

where $T_{z_{i}}\left(z_{i}\right), I_{z_{i}}\left(z_{i}\right)$ and $F_{z_{i}}\left(z_{i}\right)$ are truth membership function indeterminacy membership function ,falsity membership function of neutrosophic decision set respectively. 
Now the neutrosophic goal programming (NGP) in model (2) can be represented by crisp programming model using truth membership, indeterminacy membership, and falsity membership functions as

Maximize $\alpha$, Maximize $\gamma$, Minimize $\beta$

$T_{z_{i}}\left(z_{i}\right) \geq \alpha, i=1,2, \ldots, k$

$I_{z_{i}}\left(z_{i}\right) \geq \gamma, i=1,2, \ldots, k$

$F_{z_{i}}\left(z_{i}\right) \leq \beta, i=1,2, \ldots, k$

$z_{i} \leq t_{i}, i=1,2, \ldots \ldots, k$

$0 \leq \alpha+\beta+\gamma \leq 3$

$\alpha, \gamma \geq 0, \beta \leq 1$;

$g_{j}(x) \leq b_{j}, j=1,2, \ldots ., m$

$x_{i} \geq 0, i=1,2, \ldots, n$

\subsection{Generalized Neutrosophic Goal Programming}

The generalized neutrosophic goal programming can be formulated as

Maximize $T_{z_{i}}\left(z_{i}\right), i=1,2, \ldots ., k$

Maximize $I_{z_{i}}\left(z_{i}\right), i=1,2, \ldots ., k$

Minimize $F_{z_{i}}\left(z_{i}\right), i=1,2, \ldots, k$

Subject to

$0 \leq T_{z_{i}}\left(z_{i}\right)+I_{z_{i}}\left(z_{i}\right)+F_{z_{i}}\left(z_{i}\right) \leq w_{1}+w_{2}+w_{3}, i=1,2, \ldots, k$

$T_{z_{i}}\left(z_{i}\right) \geq 0, I_{z_{i}}\left(z_{i}\right) \geq 0, F_{z_{i}}\left(z_{i}\right) I=1,2, \ldots, k$

$T_{z_{i}}\left(z_{i}\right) \geq I_{z_{i}}\left(z_{i}\right), I=1,2, \ldots ., k$

$T_{z_{i}}\left(z_{i}\right) \geq F_{z_{i}}\left(z_{i}\right), i=1,2, \ldots, k$

$0 \leq w_{1}+w_{2}+w_{3} \leq 3$

$w_{1}, w_{2}, w_{3} \in[0,1]$

$g_{j}(x) \leq b_{j}, j=1,2, \ldots ., m$

$x_{i} \geq 0, i=1,2, \ldots ., n$

Equivalently

Maximize $\alpha$, Maximize $\gamma$, Minimize $\beta$

$T_{z_{i}}\left(z_{i}\right) \geq \alpha, i=1,2, \ldots, k$

$I_{z_{i}}\left(z_{i}\right) \geq \gamma, i=1,2, \ldots, k$

$F_{z_{i}}\left(z_{i}\right) \leq \beta, i=1,2, \ldots, k$ 


$$
\begin{aligned}
& z_{i} \leq t_{i}, i=1,2, \ldots . ., k \\
& 0 \leq \alpha+\beta+\gamma \leq w_{1}+w_{2}+w_{3} ; \\
& \alpha \in\left[0, w_{1}\right], \gamma \in\left[0, w_{2}\right], \beta \in\left[0, w_{3}\right] \\
& w_{1} \in[0,1], w_{2} \in[0,1], w_{3} \in[0,1] ; \\
& 0 \leq w_{1}+w_{2}+w_{3} \leq 3 ; \\
& g_{j}(x) \leq b_{j}, j=1,2, \ldots . ., m \\
& x_{j} \geq 0, \quad j=1,2, \ldots ., n
\end{aligned}
$$

Equivalently

Maximize $\alpha$, Maximize $\gamma$, Minimize $\beta$

$$
\begin{aligned}
& z_{i} \leq t_{i}+a_{i}\left(1-\frac{\alpha}{w_{1}}\right), i=1,2, \ldots, k \\
& z_{i} \geq t_{i}+\frac{d_{i}}{w_{2}} \gamma, i=1,2, \ldots, k \\
& z_{i} \leq t_{i}+a_{i}-\frac{\gamma}{w_{2}}\left(a_{i}-d_{i}\right), i=1,2, \ldots, k \\
& z_{i} \leq t_{i}+\frac{c_{i}}{w_{3}} \beta, i=1,2, \ldots, k \\
& z_{i} \leq t_{i}, i=1,2, \ldots ., k \\
& 0 \leq \alpha+\beta+\gamma \leq w_{1}+w_{2}+w_{3} ; \\
& \alpha \in\left[0, w_{1}\right], \gamma \in\left[0, w_{2}\right], \beta \in\left[0, w_{3}\right] ; \\
& w_{1} \in[0,1], w_{2} \in[0,1], w_{3} \in[0,1] ; \\
& 0 \leq w_{1}+w_{2}+w_{3} \leq 3 ;
\end{aligned}
$$

With the help of generalized truth, indeterminacy, falsity membership function the generalized neutrosophic goal programming based on arithmetic aggregation operator can be formulated as

$$
\text { Minimize }\left\{\frac{(1-\alpha)+\beta+(1-\gamma)}{3}\right\}
$$

Subjected to same constraints as (5)

With the help of generalized truth, indeterminacy, falsity membership function the generalized neutrosophic goal programming based on geometric aggregation operator can be formulated as

$$
\text { Minimize } \sqrt[3]{(1-\alpha) \beta(1-\gamma)}
$$

Subjected to same constraints as (5) 
Now this non-linear programming problem (5 or 6 or 7) can be easily solved by an appropriate mathematical programming to give solution of multi-objective non-linear programming problem (1) by generalized neutrosophic goal optimization approach.

\section{SOLUTION OF MULTI-OBJECTIVE WELDED BEAM OPTIMIZATION PROBLEM (MOWBP) BY GENERALIZED NEUTROSOPHIC GOAL OPTIMIZATION TECHNIQUE}

The multi-objective neutrosophic fuzzy structural model can be expressed as

Minimize $C(X)$ with target value $C_{0}$, truth tolerance $a_{C}$, indeterminacy tolerance

$d_{C}$ and rejection tolerance $c_{C}$

minimize $\delta(X)$ with target value $\delta_{0}$, truth tolerance $a_{\delta_{0}}$, indeterminacy tolerance $d_{\delta_{0}}$ and rejection tolerance $c_{\delta_{0}}$

subject to $\sigma(X) \leq[\sigma]$

$x_{i}^{\min } \leq x_{i} \leq x_{i}^{\max }$

where $X=\left[x_{1}, x_{2}, \ldots ., x_{n}\right]^{T}$ are the design variables, $\mathrm{n}$ is the group number of design variables for the welded beam design.

To solve this problem we first calculate truth ,indeterminacy and falsity membership function of objective as follows

$$
\begin{aligned}
& T_{C}^{w_{1}}(C(X))=\left\{\begin{array}{cl}
w_{1} & \text { if } C(X) \leq C_{0} \\
w_{1}\left(\frac{C_{0}+a_{C}-C(X)}{a_{C}}\right) & \text { if } C_{0} \leq C(X) \leq C_{0}+a_{C} \\
0 & \text { if } C(X) \geq C_{0}+a_{C}
\end{array}\right. \\
& I_{C(X)}^{w_{2}}(C(X))=\left\{\begin{array}{cl}
0 & \text { if } C(X) \leq C_{0} \\
w_{2}\left(\frac{C(X)-C_{0}}{d_{C}}\right) & \text { if } C_{0} \leq C(X) \leq C_{0}+a_{C} \\
w_{2}\left(\frac{C_{0}+a_{C}-C(X)}{\left.a_{C}-d_{C}\right)}\right) & \text { if } C_{0}+d_{C} \leq C(X) \leq C_{0}+a_{C} \\
0 & \text { if } C(X) \geq C_{0}+a_{C}
\end{array}\right.
\end{aligned}
$$

where $d_{C}=\frac{w_{1}}{\frac{w_{1}}{a_{C}}+\frac{w_{2}}{c_{C}}}$ 


$$
F_{C(X)}^{w_{3}}(C(X))=\left\{\begin{array}{cl}
0 & \text { if } C(X) \leq C_{0} \\
w_{3}\left(\frac{C(X)-C_{0}}{c_{C}}\right) & \text { if } C_{0} \leq C(X) \leq C_{0}+c_{C} \\
w_{3} & \text { if } C(X) \geq C_{0}+c_{C}
\end{array}\right.
$$

And

$$
\begin{aligned}
& T_{\delta(X)}^{w_{1}}(\delta(X))=\left\{\begin{array}{cl}
w_{1} & \text { if } \delta(X) \leq \delta_{0} \\
w_{1}\left(\frac{\delta_{0}+a_{\delta_{0}}-\delta(X)}{a_{\delta_{0}}}\right) & \text { if } \delta_{0} \leq \delta(X) \leq \delta_{0}+a_{\delta_{0}} \\
0 & \text { if } \delta(X) \geq \delta_{0}+a_{\delta_{0}}
\end{array}\right. \\
& I_{\delta(X)}^{w_{2}}(\delta(X))=\left\{\begin{array}{cl}
0 & \text { if } \delta(X) \leq \delta_{0} \\
w_{2}\left(\frac{\delta(X)-\delta_{0}}{d_{\delta}}\right) & \text { if } \delta_{0} \leq \delta(X) \leq \delta_{0}+a_{\delta} \\
w_{2}\left(\frac{\delta_{0}+a_{\delta}-W T(X)}{a_{\delta}-d_{\delta}}\right) & \text { if } \delta_{0}+d_{\delta} \leq \delta(X) \leq \delta_{0}+a_{\delta} \\
0 & \text { if } \delta(X) \geq \delta_{0}+a_{\delta}
\end{array}\right. \\
& d_{\delta}=\frac{w_{1}}{\frac{w_{1}}{a_{\delta}}+\frac{w_{2}}{c_{\delta}}}
\end{aligned}
$$$$
F_{\delta(X)}^{w_{3}}(\delta(X))=\left\{\begin{array}{cl}
0 & \text { if } \delta(X) \leq \delta_{0} \\
w_{3}\left(\frac{\delta(X)-\delta_{0}}{c_{\delta}}\right) & \text { if } \delta_{0} \leq \delta(X) \leq \delta_{0}+c_{\delta} \\
w_{3} & \text { if } \delta(X) \geq \delta_{0}+c_{\delta}
\end{array}\right.
$$

According to generalized neutrosophic goal optimization technique using truth, indeterminacy and falsity membership function ,MOSOP (8) can be formulated as

\section{Model -I}

Maximize $\alpha$, Maximize $\gamma$, Minimize $\beta$

$$
\begin{aligned}
& C(X) \leq C_{0}+a_{C}\left(1-\frac{\alpha}{w_{1}}\right), \\
& C(X) \geq C_{0}+\frac{d_{C}}{w_{2}} \gamma,
\end{aligned}
$$




$$
\begin{aligned}
& C(X) \leq C_{0}+a_{C}-\frac{\gamma}{w_{2}}\left(a_{C}-d_{C}\right), \\
& C(X) \leq C_{0}+\frac{c_{C}}{w_{3}} \beta, \\
& C(X) \leq C_{0}, \\
& \delta(X) \leq \delta_{0}+a_{\delta}\left(1-\frac{\alpha}{w_{1}}\right), \\
& \delta(X) \geq \delta_{0}+\frac{d_{\delta}}{w_{2}} \gamma, \\
& \delta(X) \leq \delta_{0}+a_{\delta}-\frac{\gamma}{w_{2}}\left(a_{\delta}-d_{\delta}\right), \\
& \delta(X) \leq \delta_{0}+\frac{c_{\delta}}{w_{3}} \beta, \\
& \delta(X) \leq \delta_{0}, \\
& 0 \leq \alpha+\beta+\gamma \leq w_{1}+w_{2}+w_{3} ; \\
& \alpha \in\left[0, w_{1}\right], \gamma \in\left[0, w_{2}\right], \beta \in\left[0, w_{3}\right] ; \\
& w_{1} \in[0,1], w_{2} \in[0,1], w_{3} \in[0,1] ; \\
& 0 \leq w_{1}+w_{2}+w_{3} \leq 3 ; \\
& \sigma_{i}(X) \leq[\sigma], i=1,2, \ldots . ., m \\
& x_{j} \geq 0, j=1,2, \ldots ., n \\
& W i h \in 0,
\end{aligned}
$$

With the help of generalized truth, indeterminacy, falsity membership function the generalized neutrosophic goal programming based on arithmetic aggregation operator can be formulated as

\section{Model -II}

Minimize $\left\{\frac{(1-\alpha)+\beta+(1-\gamma)}{3}\right\}$

Subjected to same constraint as Model I

With the help of generalized truth, indeterminacy, falsity membership function the generalized neutrosophic goal programming based on geometric aggregation operator can be formulated as

\section{Model -III}

Minimize $\sqrt[3]{(1-\alpha) \beta(1-\gamma)}$ 
Subjected to same constraint as Model I

Now these non-linear programming Model-I,II,III can be easily solved through an appropriate mathematical programming to give solution of multi-objective non-linear programming problem (8) by generalized neutrosophic goal optimization approach.

\section{NUMERICAL ILLUSTRATION}

A welded beam (Ragsdell and Philips 1976,Fig. 2) has to be designed at minimum cost whose constraints are shear stress in weld $(\tau)$,bending stress in the beam $(\sigma)$ ,buckling load on the bar $(P)$, and deflection of the beam $(\delta)$. The design variables are $\left[\begin{array}{l}x_{1} \\ x_{2} \\ x_{3} \\ x_{4}\end{array}\right]=\left[\begin{array}{l}h \\ l \\ t \\ b\end{array}\right]$ where $h$ is the the weld size, $l$ is the length of the weld, $t$ is the depth of the welded beam, $b$ is the width of the welded beam.

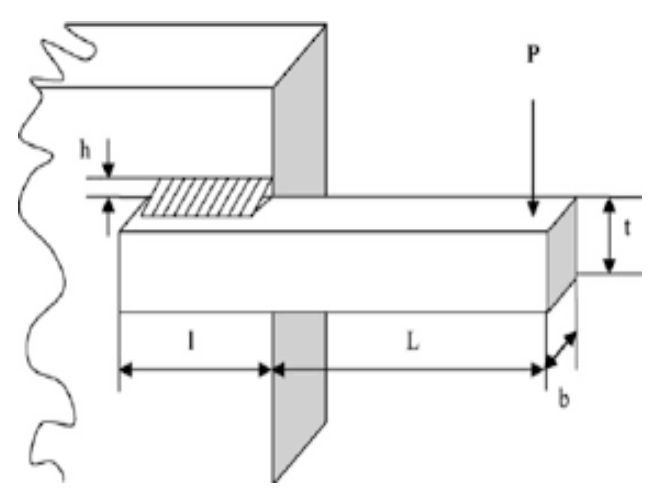

Fig.2. Design of the welded beam

\section{Cost Function}

The performance index appropriate to this design is the cost of weld assembly. The major cost components of such an assembly are (i) set up labour cost, (ii) welding labour cost, (iii) material cost.

$C(X) \equiv C_{0}+C_{1}+C_{2}$ where, $f(X)=$ cost function; $C_{0}=$ set up cost; $C_{1}=$ welding labour cost; $C_{2}=$ material cost;

Set up cost $C_{0}$ : The company has chosen to make this component a weldment, because of the existence of a welding assembly line. Furthermore, assume that 
fixtures for set up and holding of the bar during welding are readily available. The cost $C_{0}$ can therefore be ignored in this particular total cost model.

Welding labour cost $C_{1}$ : Assume that the welding will be done by machine at a total cost of $\$ 10 / \mathrm{hr}$ (including operating and maintenance expense). Furthermore suppose that the machine can lay down a cubic inch of weld in $6 \mathrm{~min}$. The labour cost is then

$$
C_{1}=\left(10 \frac{\$}{\mathrm{hr}}\right)\left(\frac{1}{60} \frac{\$}{\mathrm{~min}}\right)\left(6 \frac{\mathrm{min}}{\mathrm{in^{3 }}}\right) V_{w}=1\left(\frac{\$}{i n^{3}}\right) V_{w} . \text { Where } V_{w}=\text { weld volume, } \mathrm{in}^{3}
$$

Material cost $C_{2}: C_{2}=C_{3} V_{w}+C_{4} V_{B}$. Where $C_{3}=$ cost per volume per weld material. $\$ / \mathrm{in}^{3}=(0.37)(0.283) \quad ; \quad C_{4}=$ cost per volume of bar stock. $\$ / \mathrm{in}^{3}$ $=(0.37)(0.283) ; V_{B}=$ volume of bar,in ${ }^{3}$.From geometry $V_{w}=h^{2} l ;$ volume of the weld material $\left(\mathrm{in}^{3}\right) \quad V_{\text {weld }}=x_{1}^{2} x_{2}$ and $V_{B}=t b(L+l)$; volume of bar $\left(\mathrm{in}^{3}\right)$ $V_{b a r}=x_{3} x_{4}\left(L+x_{2}\right)$ Therefore cost function become

$$
C(X)=h^{2} l+C_{3} h^{2} l+C_{4} t b(L+l)=1.10471 x_{1}^{2} x_{2}+0.04811 x_{3} x_{4}\left(14.0+x_{2}\right)
$$

\section{Engineering Relationship}

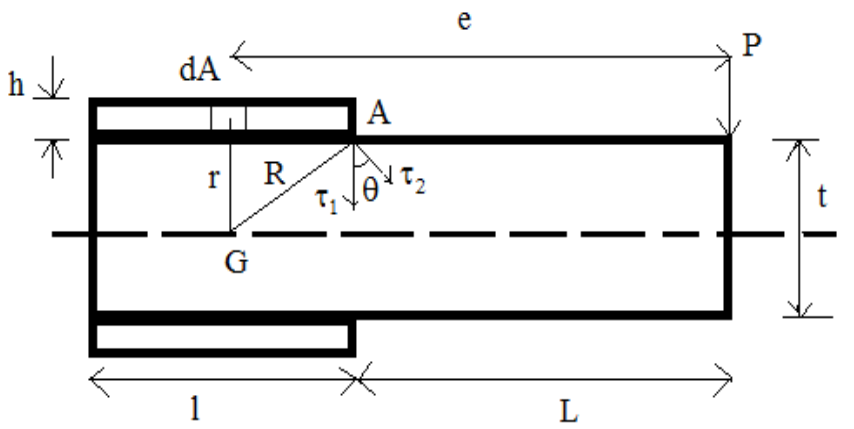

Fig 3. Shear stresses in the weld group.

\section{Maximum shear stress in weld group:}

To complete the model it is necessary to define important stress states

Direct or primary shear stress $\tau_{1}=\frac{\text { Load }}{\text { Throat area }}=\frac{P}{A}=\frac{P}{\sqrt{2} h l}=\frac{P}{\sqrt{2} x_{1} x_{2}}$

Since the shear stress produced due to turning moment $M=P . e$ at any section is proportional to its radial distance from centre of gravity of the joint ' $G$ ', therefore 
stress due to $M$ is proportional to $R$ and is in a direction at right angles to $R$. In other words $\frac{\tau_{2}}{R}=\frac{\tau}{r}=$ constant. Therefore $R=\sqrt{\left(\frac{l}{2}\right)^{2}+\left(\frac{h+t}{2}\right)^{2}}=\sqrt{\frac{x_{2}^{2}}{4}+\frac{\left(x_{1}+x_{3}\right)^{2}}{4}}$

Where, $\tau_{2}$ is the shear stress at the maximum distance $R$ and $\tau$ is the shear stress at any distance $r$. Consider a small section of the weld having area $d A$ at a distance $r$ from ' $G$ '. Therefore shear force on this small section $=\tau \times d A$ and turning moment of the shear force about centre of gravity $d M=\tau \times d A \times r=\frac{\tau_{2}}{R} \times d A \times r^{2}$. Therefore total turning moment over the whole weld area $M=\frac{\tau_{2}}{R} \int d A \times r^{2}=\frac{\tau_{2}}{R} J$. where $J=$ polar moment of inertia of the weld group about centre of gravity. Therefore shear stress due to the turning moment i.e. secondary shear stress, $\tau_{2}=\frac{M R}{J}$. In order to find the resultant stress, the primary and secondary shear stresses are combined vectorially. Therefore the maximum resultant shear stress that will be produced at the weld group, $\tau=\sqrt{\tau_{1}^{2}+\tau_{2}^{2}+2 \tau_{1} \tau_{2} \cos \theta}$, where, $\theta=$ Angle between $\tau_{1}$ and $\tau_{2}$.As $\cos \theta=\frac{l / 2}{R}=\frac{x_{2}}{2 R} ; \tau=\sqrt{\tau_{1}^{2}+\tau_{2}^{2}+2 \tau_{1} \tau_{2} \frac{x_{2}}{2 R}}$.

Now the polar moment of inertia of the throat area $(A)$ about the centre of gravity is obtained by parallel axis theorem,

$$
J=2\left[I_{x x}+A+x^{2}\right]=2\left[\frac{A \times l^{2}}{12}+A \times x^{2}\right]=2 A\left(\frac{l^{2}}{12}+x^{2}\right)=2\left\{\sqrt{2} x_{1} x_{2}\left[\frac{x_{2}^{2}}{12}+\frac{\left(x_{1}+x_{3}\right)^{2}}{2}\right]\right\}
$$

Where, $A=$ throat area $=\sqrt{2} x_{1} x_{2}, l=$ Length of the weld, $x=$ Perpendicular distance between two parallel axes $=\frac{t}{2}+\frac{h}{2}=\frac{x_{1}+x_{3}}{2}$.

Maximum bending stress in beam:

Now Maximum bending moment $=P L$, Maximum bending stress $=\frac{T}{Z}$, where $T=P L$ 
$Z=$ section modulus $=\frac{I}{y} ; I=$ moment of inertia $=\frac{b t^{3}}{12} ; y=$ distance of extreme fibre from centre of gravity of cross section $=\frac{t}{2}$; Therefore $Z=\frac{b t^{2}}{6}$. So bar bending stress $\sigma(x)=\frac{T}{Z}=\frac{6 P L}{b t^{2}}=\frac{6 P L}{x_{4} x_{3}^{2}}$.

\section{Maximum deflection in beam:}

Maximum deflection at cantilever tip $=\frac{P L^{3}}{3 E I}=\frac{P L^{3}}{3 E \frac{b t^{3}}{12}}=\frac{4 P L^{3}}{E b t^{3}}$

\section{Buckling load of beam:}

buckling load can be approximated by $P_{C}(x)=\frac{4.013 \sqrt{E I C}}{l^{2}}\left(1-\frac{a}{l} \sqrt{\frac{E l}{C}}\right)$

where, $I=$ moment of inertia $=\frac{b t^{3}}{12} ;$ torsional rigidity $C=G J=\frac{1}{3} t b^{3} G ; l=L ; a=\frac{t}{2}$;

$$
=\frac{4.013 \sqrt{E \frac{t^{2} b^{6}}{36}}}{L^{2}}\left(1-\frac{t}{2 L} \sqrt{\frac{E}{4 G}}\right)=\frac{4.013 \sqrt{E G x_{3}^{6} x_{4}^{6} / 36}}{L^{2}}\left(1-\frac{x_{3}}{2 L} \sqrt{\frac{E}{4 G}}\right) ;
$$

The single-objective optimization problem can be stated as follows

Minimize $C(X) \equiv 1.10471 x_{1}^{2} x_{2}+0.04811\left(14+x_{2}\right) x_{3} x_{4}$

Minimize $\delta(x) \equiv \frac{4 P L^{3}}{E x_{4} x_{3}^{2}}$

Such that

$$
\begin{aligned}
& g_{1}(x) \equiv \tau(x)-\tau_{\max } \leq 0 ; \\
& g_{2}(x) \equiv \sigma(x)-\sigma_{\max } \leq 0 ; \\
& g_{3}(x) \equiv x_{1}-x_{4} \leq 0 ;
\end{aligned}
$$


$g_{4}(x) \equiv 0.10471 x_{1}^{2} x_{2}+0.04811 x_{3} x_{4}\left(14+x_{2}\right)-5 \leq 0$

$g_{5}(x) \equiv 0.125-x_{1} \leq 0$

$g_{6}(x) \equiv \delta(x)-\delta_{\max } \leq 0$

$g_{7}(x) \equiv P-P_{C}(x) \leq 0$

$0.1 \leq x_{1}, x_{4} \leq 2.0$

$0.1 \leq x_{2}, x_{3} \leq 2.0$

where $\quad \tau(x)=\sqrt{\tau_{1}^{2}+2 \tau_{1} \tau_{2} \frac{x_{2}}{2 R}+\tau_{2}^{2}} \quad ; \quad \tau_{1}=\frac{P}{\sqrt{2} x_{1} x_{2}} ; \tau_{2}=\frac{M R}{J} ; M=P\left(L+\frac{x_{2}}{2}\right)$;

$R=\sqrt{\frac{x_{2}^{2}}{4}+\left(\frac{x_{1}+x_{3}}{2}\right)^{2}} ; J=\left\{\frac{x_{1} x_{2}}{\sqrt{2}}\left[\frac{x_{2}^{2}}{12}+\left(\frac{x_{1}+x_{3}}{2}\right)^{2}\right]\right\} ; \sigma(x)=\frac{6 P L}{x_{4} x_{3}^{2}} ; \delta(x)=\frac{4 P L^{3}}{E x_{4} x_{3}^{2}} ;$

$P_{C}(x)=\frac{4.013 \sqrt{E G x_{3}^{6} x_{4}^{6} / 36}}{L^{2}}\left(1-\frac{x_{3}}{2 L} \sqrt{\frac{E}{4 G}}\right) ; \quad P=$ Force on beam ; $L=$ Beam length beyond weld; $x_{1}=$ Height of the welded beam; $x_{2}=$ Length of the welded beam; $x_{3}=$ Depth of the welded beam; $x_{4}=$ Width of the welded beam; $\tau(x)=$ Design shear stress; $\sigma(x)=$ Design normal stress for beam material; $M=$ Moment of $P$ about the centre of gravity of the weld,$J=$ Polar moment of inertia of weld group; $G=$ Shearing modulus of Beam Material; $E=$ Young modulus; $\tau_{\max }=$ Design Stress of the weld; $\sigma_{\max }=$ Design normal stress for the beam material; $\delta_{\max }=$ Maximum deflection; $\tau_{1}=$ Primary stress on weld throat. $\tau_{2}=$ Secondary torsional stress on weld. Input data are given in table 1.

Table 1: Input data for crisp model (9)

\begin{tabular}{|c|c|c|c|c|c|c|}
\hline $\begin{array}{c}\text { Applied } \\
\text { load } P \\
(l b)\end{array}$ & $\begin{array}{c}\text { Beam } \\
\text { length } \\
\text { beyond } \\
\text { weld } L \\
(\mathrm{in})\end{array}$ & $\begin{array}{c}\text { Young } \\
\text { Modulus } \\
\mathrm{E} \\
(\mathrm{psi})\end{array}$ & $\begin{array}{c}\text { Value of } \\
G \\
(p s i)\end{array}$ & $\begin{array}{c}\text { Maximum } \\
\text { allowable } \\
\text { shear } \\
\text { stress } \tau_{\max } \\
(p s i)\end{array}$ & $\begin{array}{c}\text { Maximum } \\
\text { allowable } \\
\text { normal stress } \\
\sigma_{\max } \\
(p s i)\end{array}$ & $\begin{array}{c}\text { Maximum } \\
\text { allowable } \\
\text { deflection } \\
\delta_{\max } \\
(\mathrm{in})\end{array}$ \\
\hline 6000 & 14 & $3 \times 10^{6}$ & $12 \times 10^{6}$ & $\begin{array}{c}13600 \\
\text { with fuzzy } \\
\text { region } \\
50\end{array}$ & $\begin{array}{c}30000 \\
\text { with fuzzy } \\
\text { region } \\
50\end{array}$ & $\begin{array}{c}0.25 \\
\text { with fuzzy } \\
\text { region } \\
0.05\end{array}$ \\
\hline
\end{tabular}


This multi objective structural model can be expressed as neutrosophic fuzzy model as

Minimize $C(X) \equiv 1.10471 x_{1}^{2} x_{2}+0.04811\left(14+x_{2}\right) x_{3} x_{4}$ with target value 3.39 ,truth

tolerance 5 ,indeterminacy tolerance $\frac{w_{1}}{0.2 w_{1}+0.14 w_{2}}$ and rejection tolerance 7

Minimize $\delta(x) \equiv \frac{4 P L^{3}}{E x_{4} x_{3}^{2}}$; with target value 0.20 ,truth tolerance 0.23 ,indeterminacy

tolerance $\frac{w_{1}}{4.34 w_{1}+4.16 w_{2}}$ and rejection tolerance 0.24

Subject to

$g_{1}(x) \equiv \tau(x)-\tau_{\max } \leq 0 ;$

$g_{2}(x) \equiv \sigma(x)-\sigma_{\max } \leq 0$

$g_{3}(x) \equiv x_{1}-x_{4} \leq 0$

$g_{4}(x) \equiv 0.10471 x_{1}^{2} x_{2}+0.04811 x_{3} x_{4}\left(14+x_{2}\right)-5 \leq 0$

$g_{5}(x) \equiv 0.125-x_{1} \leq 0$

$g_{6}(x) \equiv \delta(x)-\delta_{\max } \leq 0$

$g_{7}(x) \equiv P-P_{C}(x) \leq 0$

$0.1 \leq x_{1}, x_{4} \leq 2.0$

$0.1 \leq x_{2}, x_{3} \leq 2.0$

where $\tau(x)=\sqrt{\tau_{1}^{2}+2 \tau_{1} \tau_{2} \frac{x_{2}}{2 R}+\tau_{2}^{2}} ; \tau_{1}=\frac{P}{\sqrt{2} x_{1} x_{2}} ; \tau_{2}=\frac{M R}{J} ; M=P\left(L+\frac{x_{2}}{2}\right)$;

$R=\sqrt{\frac{x_{2}^{2}}{4}+\left(\frac{x_{1}+x_{3}}{2}\right)^{2}} ; J=\left\{\frac{x_{1} x_{2}}{\sqrt{2}}\left[\frac{x_{2}^{2}}{12}+\left(\frac{x_{1}+x_{3}}{2}\right)^{2}\right]\right\} ; \sigma(x)=\frac{6 P L}{x_{4} x_{3}^{2}} ; \delta(x)=\frac{4 P L^{3}}{E x_{4} x_{3}^{2}} ;$

$P_{C}(x)=\frac{4.013 \sqrt{E G x_{3}^{6} x_{4}^{6} / 36}}{L^{2}}\left(1-\frac{x_{3}}{2 L} \sqrt{\frac{E}{4 G}}\right)$;

According to generalized neutrosophic goal optimization technique using truth, indeterminacy and falsity membership function ,MOWBP (10) can be formulated as 


\section{Model -I}

Maximize $\alpha$, Maximize $\gamma$, Minimize $\beta$

$$
\begin{aligned}
& 1.10471 x_{1}^{2} x_{2}+0.04811\left(14+x_{2}\right) x_{3} x_{4} \leq 3.39+5\left(1-\frac{\alpha}{w_{1}}\right), \\
& 1.10471 x_{1}^{2} x_{2}+0.04811\left(14+x_{2}\right) x_{3} x_{4} \geq 3.39+\frac{w_{1}}{w_{2}\left(0.2 w_{1}+0.14 w_{2}\right)} \gamma \\
& 1.10471 x_{1}^{2} x_{2}+0.04811\left(14+x_{2}\right) x_{3} x_{4} \leq 3.39+5-\frac{\gamma}{w_{2}}\left(2-\frac{w_{1}}{\left(0.2 w_{1}+0.14 w_{2}\right)}\right) \text {, } \\
& 1.10471 x_{1}^{2} x_{2}+0.04811\left(14+x_{2}\right) x_{3} x_{4} \leq 3.39+\frac{7}{w_{3}} \beta, \\
& 1.10471 x_{1}^{2} x_{2}+0.04811\left(14+x_{2}\right) x_{3} x_{4} \leq 3.39 \text {, } \\
& \frac{4 P L^{3}}{E x_{4} x_{3}^{2}} \leq 0.20+0.23\left(1-\frac{\alpha}{w_{1}}\right) \text {, } \\
& \frac{4 P L^{3}}{E x_{4} x_{3}^{2}} \geq 0.20+\frac{w_{1}}{w_{2}\left(4.3 w_{1}+4.1 w_{2}\right)} \gamma, \\
& \frac{4 P L^{3}}{E x_{4} x_{3}^{2}} \leq 0.20+0.23-\frac{\gamma}{w_{2}}\left(0.23-\frac{w_{1}}{\left(4.3 w_{1}+4.1 w_{2}\right)}\right) \text {, } \\
& \frac{4 P L^{3}}{E x_{4} x_{3}^{2}} \leq 0.20+\frac{0.24}{w_{3}} \beta, \\
& \frac{4 P L^{3}}{E x_{4} x_{3}^{2}} \leq 0.20 \\
& 0 \leq \alpha+\beta+\gamma \leq w_{1}+w_{2}+w_{3} ; \\
& \alpha \in\left[0, w_{1}\right], \gamma \in\left[0, w_{2}\right], \beta \in\left[0, w_{3}\right] \text {; } \\
& w_{1} \in[0,1], w_{2} \in[0,1], w_{3} \in[0,1] \text {; } \\
& 0 \leq w_{1}+w_{2}+w_{3} \leq 3 \\
& g_{1}(x) \equiv \tau(x)-\tau_{\max } \leq 0 \text {; } \\
& g_{2}(x) \equiv \sigma(x)-\sigma_{\max } \leq 0 \text {; } \\
& g_{3}(x) \equiv x_{1}-x_{4} \leq 0 \\
& g_{4}(x) \equiv 0.10471 x_{1}^{2} x_{2}+0.04811 x_{3} x_{4}\left(14+x_{2}\right)-5 \leq 0 ;
\end{aligned}
$$


$g_{5}(x) \equiv 0.125-x_{1} \leq 0$

$g_{6}(x) \equiv \delta(x)-\delta_{\max } \leq 0$

$g_{7}(x) \equiv P-P_{C}(x) \leq 0$

$0.1 \leq x_{1}, x_{4} \leq 2.0$

$0.1 \leq x_{2}, x_{3} \leq 2.0$

where $\tau(x)=\sqrt{\tau_{1}^{2}+2 \tau_{1} \tau_{2} \frac{x_{2}}{2 R}+\tau_{2}^{2}} ; \tau_{1}=\frac{P}{\sqrt{2} x_{1} x_{2}} ; \tau_{2}=\frac{M R}{J} ; M=P\left(L+\frac{x_{2}}{2}\right)$;

$R=\sqrt{\frac{x_{2}^{2}}{4}+\left(\frac{x_{1}+x_{3}}{2}\right)^{2}} ; J=\left\{\frac{x_{1} x_{2}}{\sqrt{2}}\left[\frac{x_{2}^{2}}{12}+\left(\frac{x_{1}+x_{3}}{2}\right)^{2}\right]\right\} ; \sigma(x)=\frac{6 P L}{x_{4} x_{3}^{2}} ; \delta(x)=\frac{4 P L^{3}}{E x_{4} x_{3}^{2}} ;$

$P_{C}(x)=\frac{4.013 \sqrt{E G x_{3}^{6} x_{4}^{6} / 36}}{L^{2}}\left(1-\frac{x_{3}}{2 L} \sqrt{\frac{E}{4 G}}\right)$;

With the help of generalized truth, indeterminacy, falsity membership function the generalized neutrosophic goal programming problem (10)based on arithmetic aggregation operator can be formulated as

Model -II

Minimize $\left\{\frac{(1-\alpha)+\beta+(1-\gamma)}{3}\right\}$

subjected to same constraints as (11)

With the help of generalized truth, indeterminacy, falsity membership function the generalized neutrosophic goal programming problem (10) based on geometric aggregation operator can be formulated as

\section{Model -III}

Minimize $\sqrt[3]{(1-\alpha) \beta(1-\gamma)}$

subjected to same constraints as (11)

Now these non-linear programming problem Model-I,II,III can be easily solved by an appropriate mathematical programming to give solution of multi-objective non-linear programming problem (10) by generalized neutrosophic goal optimization approach and the results are shown in the table 1 is given in table 2.Again value of membership function in GNGP technique for MOWBP (9) based on different Aggregation is given in Table 3. 
Table 2: Comparison of GNGP solution of MOWBP (9) based on different Aggregation

\begin{tabular}{|c|c|c|c|c|c|c|}
\hline Methods & $x_{1}$ in & $x_{2}$ in & $x_{3}$ in & $x_{4}$ in & $C(X)$ & $\delta(X)$ \\
\hline $\begin{array}{c}\text { Generalized Fuzzy Goal } \\
\text { programming(GFGP) } \\
w_{1}=0.15\end{array}$ & 1.297612 & 0.9717430 & 1.693082 & 1.297612 & 3.39 & 0.20 \\
\hline $\begin{array}{c}\text { Generalized Intuitionistic } \\
\text { Fuzzy Goal } \\
\text { programming(GIFGP) } \\
w_{1}=0.15 w_{3}=0.8\end{array}$ & 1.297612 & 0.9717430 & 1.693082 & 1.297612 & 3.39 & 0.20 \\
\hline $\begin{array}{c}\text { Generalized } \\
\text { Neutrosophic Goal } \\
\text { programming (GNGP) } \\
w_{1}=0.4, w_{2}=0.3, w_{3}=0.7\end{array}$ & 1.347503 & 0.7374240 & 2 & 1.347503 & 3.39 & 2 \\
\hline $\begin{array}{l}\text { Generalized Intuitionistic } \\
\text { Fuzzy optimization } \\
\text { (GIFGP) based on } \\
\text { Arithmetic Aggregation } \\
\quad w_{1}=0.15, w_{3}=0.8\end{array}$ & 1.297612 & 0.9717430 & 1.693082 & 1.297612 & 3.39 & 0.20 \\
\hline $\begin{array}{c}\text { Generalized Neutosophic } \\
\text { optimization (GNGP) } \\
\text { based on Arithmetic } \\
\text { Aggregation } \\
w_{1}=0.4, w_{2}=0.3, w_{3}=0.7 \\
\end{array}$ & 1.347503 & 0.7374240 & 2 & 1.347503 & 3.39 & 0.20 \\
\hline $\begin{array}{l}\text { Generalized Intuitionistic } \\
\text { Fuzzy optimization } \\
\text { (GIFGP) based on } \\
\text { Geometric Aggregation } \\
w_{1}=0.15, w_{3}=0.8 \\
\end{array}$ & 1.372 & 0.697176 & 2 & 1.37200 & 3.39 & 0.2 \\
\hline $\begin{array}{c}\text { Generalized Neutosophic } \\
\text { optimization (GNGP) } \\
\text { based on Geometric } \\
\text { Aggregation } \\
w_{1}=0.4, w_{2}=0.3, w_{3}=0.7\end{array}$ & 1.372 & 0.6971 & 2 & 1.372 & 3.39 & 0.2 \\
\hline
\end{tabular}

Here we almost same solutions for the different value of $w_{1}, w_{2}, w_{3}$ in different aggregation method for objective functions. From Table .2 it is clear that the cost of welding and deflection are almost same in fuzzy and intuitionistic fuzzy as well as neutrosophic optimization technique. Moreover it has been seen that desired value obtained in different aggregation method have not affected by variation of methods in perspective of welded beam design optimization. 


\section{CONCLUSIONS}

The research study investigates that neutrosophic goal programming can be utilized to optimize a nonlinear welded beam design problem. . The results obtained for different aggregation method of the undertaken problem show that the best result is achieved using geometric aggregation method. The concept of neutrosophic optimization technique allows one to define a degree of truth membership, which is not a complement of degree of falsity; rather, they are independent with degree of indeterminacy. As we have considered a non-linear welded beam design problem and find out minimum cost of welding of the structure as well as minimum deflection, the results of this study may lead to the development of effective neutrosophic technique for solving other model of nonlinear programming problem in different field.

Conflict of interests: The authors declare that there is no conflict of interests.

\section{ACKNOWLEDGEMENT}

The research work of Mridula Sarkar is financed by Rajiv Gandhi National Fellowship (F1-17.1/2013-14-SC-wes-42549/(SA-III/Website)), Govt of India.

TKR participated in result analysis in the manuscript. MS carried out rest of things in manuscript.

\section{REFERENCES}

[1] Zadeh, L.A. (1965). Fuzzy set. Information and Control, 8(3), 338-353.

[2] Coello, C.A.C. 2000b. Use of a self-adaptive penalty approach for engineering optimization problems. Comput. Ind., 41: 113-127. DOI: 10.1016/S01663615(99)00046-9.

[3] Reddy , M. J.; Kumar, D. N.; ,'An efficient multi-objective optimization algorithm based on swarm intelligence for engineering design' Engineering Optimization, Vol. 39, No. 1, January 2007, 49-68.

[4] Carlos A. Coello Coello, 'Solving Engineering Optimization Problems with the Simple Constrained Particle Swarm Optimizer', Informatica 32 (2008) 319-326. 
[5] Lee,K.S., Geem,Z.W. 'A new meta-heuristic algorithm for continuous engineering optimization: harmony search theory and practice' Comput. Methods Appl. Mech. Engrg. 194 (2005) 3902-3933.

[6] S. Kazemzadeh Azada, ,O. Hasançebia and O. K. Erol ' Evaluating efficiency of big-bang big-crunch algorithm in benchmark engineering optimization problems','Int. J. Optim. Civil Eng., 2011; 3:495-505.

[7] Hasançebi, O. and Azad ,S.K.' An efficient metaheuristic algorithm for engineering optimization: SOPT' ' int. j. optim. civil eng., 2012; 2(4):479-487.

[8] Mahdavi , M. , Fesanghary , M., Damangir, E. , 'An improved harmony search algorithm for solving optimization problems" Applied Mathematics and Computation 188 (2007) 1567-1579.

[9] Atanassov, K. T. (1986). Intuitionistic fuzzy sets. Fuzzy Sets and Systems,20(1), 87-96.

[10] Smarandache, F. (1995). Neutrosophy, neutrosophic probability, set and logic, Amer. Res. Press, Rehoboth, USA, 105.

[11] Shuang Li,G. and Au,S.K.' Solving constrained optimization problems via Subset Simulation''2010 4th International Workshop on Reliable Engineering Computing (REC 2010),doi:10.3850/978-981-08-5118-7 069.

[12] Yanling Wei, Jianbin Qiu, Hamid Reza Karimi, Reliable ''Output Feedback Control of Discrete-Time Fuzzy Affine Systems With Actuator Faults"' Doi: 10.1109/TCSI.2016.2605685,2016,1-12.

[13] Yanling Wei, Jianbin Qiu, Hak-Keung Lam, and Ligang Wu,' Approaches to T-S Fuzzy-Affine-Model-Based Reliable Output Feedback Control for Nonlinear It ${ }^{\wedge} \quad$ Stochastic $\quad$ Systems'"2016, $\quad$ DOI 10.1109/TFUZZ.2016.2566810,pp-1-14.

[14] Sarkar ,M .,Dey ,Samir.,Roy,T.K.,'Multi-Objective Neutrosophic Optimization Technique and its Application to Structural Design', International Journal of Computer Applications (0975 - 8887) Vol 148 No.12, August 2016.

[15] Das, P., Roy,T.K.,'Multi-objective non-linear programming problem based on Neutrosophic Optimization Technique and its application in Riser Design Problem'" Neutrosophic Sets and Systems, (88-95),Vol. 9, 2015.

[16] K. Deb, Optimal design of a welded beam via genetic algorithms, AIAA Journal 29 ,(11) 1991. 2013-2015.

[17] Deb, K., Pratap, A. and Moitra, S., Mechanical component design for multiple objectives using elitist non-dominated sorting GA. In Proceedings of the Parallel Problem Solving from Nature VI Conference,Paris, 16-20 September 2000, pp. 859-868. 
[18] K.M. Ragsdell, D.T. Phillips, Optimal design of a class of welded structures using geometric programming, ASME Journal of Engineering for Industries 98 (3)1976. 1021-1025, Series B.

[19] Charns, A., \& Cooper, R. (1961). Management Models and Industrial Application of Linear Programming.

[20] Dey, S., \& Roy, T. K. (2015). Intuitionistic Fuzzy Goal Programming Technique for Solving Non-Linear Multi-objective Structural Problem. Journal of Fuzzy Set Valued Analysis, 2015(3), 179-193. 